\title{
BMJ Open Can a simple 'cost-awareness' campaign for laparoscopic hysterectomy change the use and costs of disposable surgical supplies? Pre-post non-controlled study
}

\author{
Sue Ross (D) , ${ }^{1}$ Douglas Lier, ${ }^{1}$ Goldie Mackinnon, ${ }^{1}$ Christine Bentz, ${ }^{1}$ \\ Gloria Rakowski, ${ }^{2}$ Valerie A Capstick ${ }^{1}$
}

To cite: Ross S, Lier D, Mackinnon G, et al. Can a simple 'cost-awareness' campaign for laparoscopic hysterectomy change the use and costs of disposable surgical supplies? Pre-post non-controlled study. BMJ Open 2019;9:e027099. doi:10.1136/ bmjopen-2018-027099

- Prepublication history and additional material for this paper are available online. To view these files, please visit the journal online (http://dx.doi. org/10.1136/bmjopen-2018027099).

Received 20 0ctober 2018 Revised 23 October 2019 Accepted 14 November 2019

Check for updates

(C) Author(s) (or their employer(s)) 2019. Re-use permitted under CC BY-NC. No commercial re-use. See rights and permissions. Published by BMJ.

${ }^{1}$ Obstetrics and Gynecology, University of Alberta, Edmonton, Alberta, Canada

${ }^{2}$ Women's Health, Lois Hole Hospital for Women, Alberta Health Services, Edmonton, Alberta, Canada

Correspondence to

Dr Sue Ross; sjross@ualberta.ca

\section{ABSTRACT}

Objectives Does a cost-awareness campaign for gynaecologists lead to a change in use and costs of disposable surgical supplies for laparoscopic hysterectomy (LH) without increasing hospital utilisation measures (operating room (OR) time or hospital length of stay (LOS))? Design Pre-post non-controlled study. The OR database was used to identify relevant cases before and after the cost-awareness intervention, and provided information on quantity of each supply item, operative details and LOS. Setting Lois Hole Hospital for Women, Edmonton, Alberta, Canada.

Participants 12 laparoscopic trained gynaecologists (7 female, 5 male) participated in both phases of the study. Eligible surgical cases were all LH cases for any indication for women aged $\geq 18$ years. 201 cases were undertaken before the intervention (2011-2013) and 229 cases after the intervention (2016-2017).

Intervention The cost-awareness intervention for gynaecologists included site meetings and rounds providing information on costs of disposable and reusable instruments, a full day skills lab, OR posters about cost and effectiveness of disposable and reusable surgical supplies and demonstrations of reusable equipment (2015-2016).

Primary outcome measure Disposable supplies costs per case (standardised for 2016 unit costs).

Results There was a significant $(p<0.05)$ reduction (unadjusted) in disposable supplies cost per case for LH between cases before and after the intervention: from \$C1073, SD 281, to \$C943 SD 209. Regression analysis found that the adjusted cost per case after the intervention was \$C116 lower than before the intervention $(95 \% \mathrm{Cl}$ -160 to -71$)$. Neither OR time nor hospital LOS differed significantly between cohorts.

Conclusions Our study suggests that cost-awareness campaigns may be associated with reduction in the cost of surgery for LH. However, many other factors may have contributed to this cost reduction, possibly including other local initiatives to reduce costs and emerging evidence indicating lack of effectiveness of some surgical practices.

\section{INTRODUCTION}

Given increasing financial constraints in healthcare systems worldwide, with growing concerns about responsible stewardship
Strengths and limitations of this study

- Our study investigated disposable surgical supply costs before and after the implementation of a costawareness campaign for gynaecologists who carry out laparoscopic hysterectomy: this work adds to the surgical literature in which net costs are rarely evaluated.

- The cost-awareness intervention was a strengththe intervention incorporated several strategies including a skills lab, and operating room (OR) posters and equipment demonstrations.

- The main outcomes were cost per case of disposable surgical supplies (all costs standardised to mid2016 to avoid impact of unit cost changes over time) and hospital utilisation measures (OR time or hospital length of stay) during the index admission, for the cohorts of patients before and after the intervention.

- Data were recorded in 'real-time' in the same OR database before and after the intervention-data were complete for surgical details and devices used.

- Limitations were lack of a control group of patients and data were available only for index admissionlonger-term follow-up was not available.

of resources, ${ }^{1}$ physicians are increasingly expected to take on an additional role as guardians of resources. ${ }^{2}{ }^{3}$ This role has even been incorporated into physician training, ${ }^{4}$ and physicians are encouraged to be aware of the costs of treatment options so that they can provide cost-effective and safe treatments. ${ }^{2}$ Despite these expectations, awareness of healthcare costs is poor in a variety of surgical disciplines including gynaecology, ${ }^{5-7}$ with physicians consistently underestimating the cost of more expensive items. ${ }^{8-10}$

Published research has demonstrated that two types of intervention can reduce intraoperative costs. One effective intervention reduces costs by increasing standardisation of operative supplies. ${ }^{11}$ Providing physician feedback on their own operating room (OR) 
costs compared with their peers has also been found effective. ${ }^{11}$ Increasing individual surgeon cost-awareness is also an intervention that could be adopted locally.

At the Lois Hole Hospital for Women (LHHW), a major acute care hospital in Edmonton, Canada, variations in OR surgical costs were observed specifically for laparoscopic hysterectomy (LH). This procedure became the most common approach for hysterectomy because of perceived advantages such as excellent patient outcomes, earlier return to normal activities, shorter hospital stays and fewer wound infections. ${ }^{12-14}$ As well, increasing availability of training in minimally invasive surgery accounted for growth in the number of laparoscopic gynaecologists, with 12 (of 18) gynaecologists performing LH by 2011. Consequently, the number of LHs also increased each year, from 40 cases in 2008 to $90 \mathrm{LH}$ in 2013. Some LHHW surgeons favoured disposable laparoscopic instruments, while others preferred reusable instruments, resulting in variation in OR supply costs, perhaps as a result of lack of standardisation and lack of physician awareness of costs. We hypothesised that a cost-awareness campaign for LH incorporating exposure to reusable instruments may influence gynaecologists' choice of instruments and could reduce the costs of $\mathrm{LH}$.

\section{Research question}

Does a cost-awareness campaign for gynaecologists lead to a change in use and costs of disposable surgical supplies for LH without increasing hospital utilisation measures (OR time or hospital length of stay (LOS))?

\section{METHODS}

We used a pre and post non-controlled study design, based on two non-concurrent retrospective cohorts of surgical cases undertaken by eligible surgeons who consented to participate, to study the impact of a cost-awareness intervention campaign at LHHW. Surgical cases completed between January 2011 and August 2013 comprised the 'pre' cohort. The start of the intervention was delayed until research funding was received. The intervention was conducted from March 2015 to May 2016. Cases identified between June 2016 and May 2017 comprised the 'post' intervention cohort. Cases between September 2013 and February 2015 were not included in the study to reduce the possible impact of contamination by early exposure to any elements of the intervention which were being developed during that time.

For this analysis, we have taken the perspective of the hospital, and the overall goal was to reduce the costs of disposable surgical supplies without affecting hospital utilisation measures (OR time or hospital LOS).

\section{Data collection}

The same LHHW OR database was in use without significant changes to documentation practices during the full duration of the study (from January 2011 to May 2017) and was used to identify eligible LH cases. Data were collected on supply item use for each patient throughout the time they were in the OR including details about the type and number of each supply item used for that specific patient. This 'point of use' data entry formed part of the OR supply management and control system, and ensured that the data were as complete and accurate as possible for the purpose of this research. The database also identified the type of hysterectomy performed, and other procedure-related information including OR time. In addition, patient-specific data were collected from hospital charts, for example: age, body mass index (BMI) and data related to previous pregnancies and births, and hospital LOS of the index admission. Measures of hospital utilisation were OR time and hospital LOS. Patient follow-up was to the time of hospital discharge.

Data collection before and after the intervention was approved by the University of Alberta Health Research Ethics Board (HREB) and the consent of participating physicians (the research subjects) was obtained. The HREB provided a waiver of consent for individual patients on the basis that it would be impractical to collect individual patient consent for this study. Strict data confidentiality practices were implemented in accordance with the Alberta Health Information Act.

\section{Surgeon participants and surgical cases}

To reduce any impact of a learning curve on the study outcomes, only surgeons trained in laparoscopic surgical techniques, and who had undertaken four or more LH in the year before the intervention were invited to take part in the study. The 12 eligible surgeons who performed LH during the periods before and after the intervention all gave consent to use data related to their practice.

All surgery was performed in the LHHW Women's OR suite. The eligibility criteria for cases included: all women aged $\geq 18$ years having a LH for any indication and all types of LH procedures. Only cases starting as LH were eligible, including total laparoscopic hysterectomy (TLH), subtotal laparoscopic hysterectomy and laparoscopic-assisted vaginal hysterectomy, as well as those procedures that were converted to a total abdominal hysterectomy.

\section{Intervention}

The intervention was designed as an intensive costawareness campaign to highlight the relative cost benefit of reusable surgical supplies, and was undertaken in the first quarter of 2015. This campaign consisted of:

1. Department of Obstetrics and Gynaecology site meetings and rounds including information on costs of disposable and reusable instruments.

2. Reusable versus disposable instrument use as a topic of the Department of Obstetrics and Gynaecology Research Day keynote speaker (a leading laparoscopic surgeon, and champion of reusable instruments).

3. A full-day skills lab for surgeon participants (plus residents and OR nurses) to provide hands-on experience with reusable instruments. 
The main event of the campaign was the skills lab, but unfortunately none of the eligible surgeon participants took part in the lab. Therefore, additional cost-awareness strategies were developed and implemented in the OR in early 2016.

The additional cost-awareness strategies involved presenting three posters that were changed each month over a 3-month period. The posters presented information on cost and effectiveness of disposable and reusable surgical supplies, and were displayed in the OR and other hospital settings where gynaecologists were likely to see them (eg, clinics). Small pocket card versions of the posters were distributed to all gynaecologists over the same period. For copies of the posters, see online supplementary appendix 1 . As well, a demonstration of reusable equipment was available in the physician coffee room (with coffee and muffins) over two consecutive weeks.

The costs (in Canadian \$) of the intervention were as follows: animals for the skills lab-\$C3103, research staff time to develop, prepare and distribute the cost-awareness materials-\$C2125, investigator time developing the costawareness materials-\$C2400, printing cost for posters and pocket cards-\$C200, and coffee for the OR demonstrations- $\$$ C60. The total cost of the intervention was thus $\$ \mathrm{C} 7798$, or $\$ \mathrm{C} 34$ per case in the postintervention cohort. This value was used to estimate the net cost per case.

\section{Cost of disposable surgical supply items (primary outcome)}

A standard list of 2016 unit costs (prices) was compiled from the OR database, that included each of the disposable surgical supply items identified during the two study periods (see online supplementary appendix 2). Costs for disposable supply items that changed during 2016 were standardised to mid-2016 unit costs.

For each surgical case, the quantity used of each disposable item was multiplied by the corresponding 2016 unit cost, and the products summed to estimate the total disposable surgical supplies cost per case. Net cost per case was also calculated for the postintervention cases, taking into account the cost of the intervention itself.

\section{Statistical analysis}

A range of single variable analyses (patient characteristics, procedure-related variables and health system variables) were conducted. To test for differences between the cohorts before and after the intervention, $\chi^{2}$ tests (and Fischer's exact test where appropriate) were performed for categorical variables and t-tests for continuous variables from normal distributions. For variables with nonnormal distributions such as disposable supplies cost and quantity of supply items, bootstrap CIs were estimated (5000 replicates).

We examined the difference in mean disposable supplies cost and quantity of disposable surgical supply items used between precohort and postcohort in five subgroups of surgical items. Disposable items were grouped into five categories, based on the observed clustering pattern of their unit costs. We verified that the cost-grouping method achieved a high between-group variance in unit cost $(93 \%)$ and low within-group variance $(7 \%)$. The lowest cost group contained items with unit cost of \$C30 or less (eg, tubing, sutures), while the upper cost group comprised items of $\$ \mathrm{C} 350$ or more (eg, sealing units). For each surgical case, the cost of supply items in each unit cost group was estimated. We then compared the average cost of each group and quantity of disposable surgical supply items between study precohort and postcohort. Bootstrap CIs (5000 replicates) were estimated for differences between cohorts.

Our primary analysis was based on multiple linear regression at the patient level. A dummy variable (binary variable with $0=$ control and $1=$ intervention) was included to determine whether there was an intervention effect. The final regression model was:

Disposable cost per patient $=\beta_{1}+\beta_{2}$ (intervention dummy) $+\beta_{3}$ (non-TLH procedure dummy) $+\beta_{4-14}$ (surgeon dummies) + random error term

In addition to the intervention variable, the other explanatory variables included in the analysis were a dummy variable indicating whether the surgical procedure was other than TLH, and dummy variables for 11 surgeons. Other patient-related variables for which we collected data were not statistically significant in preliminary testing, including patient age. The non-TLH dummy variable was used rather than individual variables for the three non-TLH procedures because the number of each non-TLH procedure was very small (see Results section). Dummy variables were used for 11 of 12 surgeons to capture the disparity in the cost of disposables used by individual surgeons. The reference surgeon performed the average number of surgeries and incurred the average disposable supplies cost compared with participating surgeons.

A bootstrapping procedure (5000 replications) was used to estimate a $95 \%$ CI for each regression coefficient. All analyses were performed with R Project 2017 core software and the Rcmdr V.2.4-1 package. Data management was conducted with FileMaker Pro Advanced V.13.04.

\section{Patients and public involvement}

Neither patients nor public were involved in the design or conduct of this research about the effect of the costawareness campaign for gynaecologists.

\section{RESULTS}

Description of the surgeon sample and surgical case cohorts

The 12 surgeons who were the subjects of this study, included seven women and five men, and the year of their first LH as staff ranged between 2000 and 2012. All 12 surgeons participated in both phases of the study.

The precohort consisted of 201 cases and the postcohort included 229 cases (table 1, with missing obstetrical and hospital LOS data highlighted in column 1). Differences between study cohorts before and after the 
Table 1 Patient characteristics, surgical details and resource use for the cohorts before and after the cost-awareness intervention

\begin{tabular}{|c|c|c|c|c|}
\hline Variables & $\begin{array}{l}\text { Precohort* } \\
\mathrm{n}=201\end{array}$ & $\begin{array}{l}\text { Postcohort* } \\
n=229\end{array}$ & Diff† & $P$ value \\
\hline \multicolumn{5}{|l|}{ Patient characteristics } \\
\hline Age (years), mean (SD) & $48.3(11.8)$ & $44.1(11.7)$ & -4.2 & $<0.001$ \\
\hline BMI, mean (SD) & $28.5(6.5)$ & $30.5(6.8)$ & 2.0 & 0.002 \\
\hline Gravidity, mean (SD); before (n147); after (n228) & $2.4(1.9)$ & $2.1(1.9)$ & -0.3 & 0.201 \\
\hline Parity, mean (SD); before (n200); after (n228) & $1.7(1.3)$ & $1.6(1.4)$ & -0.1 & 0.393 \\
\hline VD, mean (SD); before (n198); after (n204) & $1.5(1.3)$ & $1.1(1.3)$ & -0.4 & 0.006 \\
\hline Nulliparous (n/\%); before (n200); after (n228) & $46(23.0)$ & $70(30.7)$ & 7.7 & 0.074 \\
\hline 21 C-section (n/\%); before (n198); after (n204) & $25(12.6)$ & $49(24.0)$ & 11.4 & 0.003 \\
\hline \multicolumn{5}{|l|}{ Surgical details } \\
\hline \multicolumn{5}{|l|}{ Type of surgical procedure } \\
\hline TLH (n/\%) & $187(93.0)$ & $220(96.1)$ & 3.1 & 0.163 \\
\hline LAVH (n/\%) & $6(3.0)$ & $4(1.7)$ & -1.3 & 0.395 \\
\hline SLH (n/\%) & $2(1.0)$ & $0(0)$ & -1.0 & 0.218 \\
\hline $\mathrm{TAH}(\mathrm{n} / \%)$ & $6(3.0)$ & $5(2.2)$ & -0.8 & 0.599 \\
\hline Non-TLH (n/\%) & $14(7.0)$ & $9(3.9)$ & -3.1 & 0.163 \\
\hline At least 1 ovary removed $(\mathrm{n} / \%)$ & $111(55.2)$ & $119(52.0)$ & -3.2 & 0.499 \\
\hline At least 1 fallopian tube removed $(n / \%)$ & $170(84.6)$ & $220(96.1)$ & 11.5 & $<0.001$ \\
\hline Duration of surgery (min), mean (SD) & $150.1(65.1)$ & $139.7(88.3)$ & -10.4 & $>0.05 \ddagger$ \\
\hline \multicolumn{5}{|l|}{ Resource use } \\
\hline LOS (days), mean (SD); before (n201); after (n204) & $1.3(0.7)$ & $1.2(0.6)$ & -0.1 & $>0.05 \ddagger$ \\
\hline Quantity of disposable items, mean (SD) & $14.2(3.4)$ & $16.5(3.5)$ & 2.3 & $\leq 0.05 \ddagger$ \\
\hline Cost $(\$ C)$ of disposable items used; mean (SD) & $1073(281)$ & 943 (209) & -130 & $\leq 0.05 \ddagger$ \\
\hline
\end{tabular}

Gravidity, number of pregnancies.

Nulliparous, never having borne a viable child.

Parity, number of viable births.

${ }^{*}$ Full sample achieved except where noted in patient characteristics (column 1).

†The differences (column 3-column 2) are unadjusted for the effect of other variables.

‡Based on a $95 \% \mathrm{Cl}$ from 5000 bootstrap replicates.

BMI, body mass index; LAVH, laparoscopic-assisted vaginal hysterectomy; LOS, length of stay; SLH, subtotal laparoscopic hysterectomy;

$\mathrm{TAH}$, total abdominal hysterectomy; TLH, total laparoscopic hysterectomy; VD, vaginal delivery-number of VD per patient.

intervention were significant in 4 of the 7 patient characteristic variables shown: patient age, BMI, number of vaginal deliveries and the proportion of women with at least one Cesarean-section. Mean parity (number of viable births), mean gravidity (number of pregnancies) and the proportion of nulliparous women (who had never borne a viable child) were did not differ significantly between precohort and postcohort.

\section{Surgical case characteristics}

The proportion of women with at least one fallopian tube removed increased significantly $(84.6 \%$ vs $96.1 \%$, $\mathrm{p}<0.001)$. The conversion rate to non-TLH was $7.0 \%$ versus $3.9 \%$ (not significantly different). The remaining surgical procedure-related variables, including the average duration of the procedure and hospital LOS, did not differ significantly (table 1 ).

\section{Disposable surgical supplies quantity and costs}

The differences between precohort and postcohort for mean quantity of disposable items used and the mean cost of disposables per surgical case were significant (table 1). The postcohort required on average $16 \%$ more disposable items $(\mathrm{p}<0.05)$ but the unadjusted mean cost of disposables was about $12 \%$ less per case $(\mathrm{p}<0.05)$, and mean net cost about $9 \%$ less per case.

\section{Surgical supplies unit cost groups: analysis of cost and quantity}

The unit cost group with the greatest number of unique supply items (distinct from the quantity of each item) is group A with $58 \%$ of the 86 devices (table 2A). In this low cost group, the average cost per surgical case for precohort and postcohort was virtually equal. Although the mean cost of group B was slightly lower for the postcohort, 
Table 2 (A) Mean cost of disposable surgical supply items by unit cost group for the cohorts before and after the costawareness intervention; (B) mean quantity of disposable surgical supply items by unit cost group for the cohorts before and after the cost-awareness intervention

\begin{tabular}{|c|c|c|c|c|c|}
\hline Unit cost group & $\begin{array}{l}\text { Items } \\
\text { N (\%) }\end{array}$ & $\begin{array}{l}\text { Before } \\
\text { cost \$C } \\
\text { mean (SD) }\end{array}$ & $\begin{array}{l}\text { After } \\
\text { cost \$C } \\
\text { mean (SD) }\end{array}$ & Diff* & $\begin{array}{l}95 \% \mathrm{Cl} \dagger \\
2.5 \% \text { to } 97.5 \%\end{array}$ \\
\hline $\mathrm{A}(\leq \$ \mathrm{C} 30$ per item $)$ & $50(58)$ & $118(26)$ & $114(31)$ & -4 & -9 to 1 \\
\hline $\mathrm{B}(>\$ \mathrm{C} 30$ and $\leq \$ \mathrm{C} 70$ per item $)$ & $10(12)$ & $100(83)$ & $93(44)$ & -7 & -21 to 5 \\
\hline$C(>\$ C 70$ and $\leq \$ C 105$ per item) & $8(9)$ & $155(90)$ & $89(75)$ & $-66 \ddagger$ & -81 to -50 \\
\hline $\mathrm{D}(>\$ \mathrm{C} 105$ and $\leq \$ \mathrm{C} 350$ per item) & $11(13)$ & $167(150)$ & $91(112)$ & $-76 \ddagger$ & -102 to -50 \\
\hline Unit cost group & $\begin{array}{l}\text { Items } \\
\text { N (\%) }\end{array}$ & $\begin{array}{l}\text { Before } \\
\text { quantity } \\
\text { mean (SD) }\end{array}$ & $\begin{array}{l}\text { After } \\
\text { quantity } \\
\text { mean (SD) }\end{array}$ & Diff* & $\begin{array}{l}95 \% \mathrm{Cl} \dagger \\
2.5 \% \text { to } 97.5 \%\end{array}$ \\
\hline $\mathrm{A}(\leq \$ \mathrm{C} 30$ per item $)$ & $50(58)$ & $8.14(1.62)$ & $11.89(3.10)$ & $3.75 \ddagger$ & 3.31 to 4.22 \\
\hline $\mathrm{B}(>\$ \mathrm{C} 30$ and $\leq \$ \mathrm{C} 70$ per item $)$ & $10(12)$ & $1.99(1.65)$ & $1.93(0.91)$ & -0.06 & -0.32 to 0.20 \\
\hline
\end{tabular}

*The differences (column 4-column 3) are unadjusted for the effect of other variables.

†Bootstrap $\mathrm{Cl}, 5000$ replicates.

$\ddagger$ The difference is statistically significant at a $95 \%$ level of confidence.

the difference was not significant. For the next two higher cost groups $(\mathrm{C}$ and $\mathrm{D})$, the mean cost per case for the postcohort was significantly less than for precohort. The higher cost group E encompasses the relatively costly sealing devices (eg, Ligasure) used almost equally in pre and post cases and consequently the average cost was only slightly higher for the postcohort.

Table 2B presents the quantity of supply items used per surgical case for each of the cost groups. After the intervention, there were significantly more lower cost items used per case compared with the precohort (group A, \$C30 or less), with fewer mid-cost items (groups C and $\mathrm{D}$, items costing between $\$ \mathrm{C} 70$ and $\$ \mathrm{C} 350$ ). The increase in the quantity of group A items more than offsets the decreases in quantities used in the mid-cost groups $(\mathrm{C}$ and $\mathrm{D}$ ), accounting for the overall increase in quantity per case shown in table 1 .

\section{Regression analysis}

None of the patient characteristic variables were significant explanatory variables in the preliminary regression analysis and were therefore not included in the final model (table 3).

The intervention effect $\left(\beta_{2}\right)$ was statistically significant indicating an average saving of \$C116 per case $(95 \%$ CI -160 to 71 ). This adjusted estimate was $11 \%$ lower than the unadjusted difference in cost of disposable items, of -\$C130 per case (table 1). The estimated coefficient of the non-TLH procedure variable indicates that the cost of disposable supplies was significantly less for those procedures. The coefficient estimates of four surgeon dummy variables were also significant indicating that the utilisation of disposable supplies was significantly different from the reference physician. The non-constant variance test result confirmed the standard assumption of constant variance in the error term.

Based on the adjusted amount of savings per case (\$C116), the intervention reaches a break-even point at 67 cases $(\$ C 7798 / \$ C 116)$. Beyond this number of cases, the intervention becomes cost-saving.

\section{DISCUSSION}

Our study identified a significant \$C116 (95\% CI -160 to 71) reduction in the cost of disposable surgical supply items per case following the introduction of a cost-awareness

Table 3 Multiple regression results

\begin{tabular}{|c|c|c|c|}
\hline Variable & $\begin{array}{l}\text { Estimated } \\
\text { coefficient }\end{array}$ & SE* $^{*}$ & $\begin{array}{l}\mathrm{Cl}+2.5 \% \text { to } \\
97.5 \%\end{array}$ \\
\hline \multicolumn{4}{|c|}{ Disposable supplies cost per case (independent variable) } \\
\hline Intercept $\left(B_{1}\right)$ & $1114.1 \ddagger$ & 34.1 & 1046.9 to 1182.1 \\
\hline $\begin{array}{l}\text { Intervention dummy } \\
\left(B_{2}\right)\end{array}$ & $-115.5 \ddagger$ & 22.2 & -159.5 to -71.1 \\
\hline $\begin{array}{l}\text { Non-TLH procedure } \\
\left(\mathrm{B}_{3}\right)\end{array}$ & $-128.2 \ddagger$ & 47.5 & -220.5 to -35.0 \\
\hline \multicolumn{4}{|c|}{4 of 11 surgeon dummy variables $\left(B_{4}-B_{14}\right)$ were significant } \\
\hline
\end{tabular}

$\mathrm{N}=430$.

Degrees of freedom: 416.

Adjusted $R^{2}=0.214$.

F-statistic $=10.0, p$ value $<0.001$.

Non-constant variance test (ncvTest): $\chi^{2}=0.266$, $p$ value $=0.606$.

*Bootstrap SE, 5000 replicates.

†Bootstrap $\mathrm{Cl}, 5000$ replicates.

$\ddagger$ Statistically significant at $95 \% \mathrm{Cl}$.

$\mathrm{TLH}$, total laparoscopic hysterectomy. 
campaign for surgeons. This reduction compares favourably to the $\$ \mathrm{C} 34$ cost of the intervention per case. The cost reduction was observed despite using standardised 2016 unit costs and an overall increase in the quantity of disposable items per case observed postintervention. This finding was explained by our observation that there was a significant increase in the use of the lowest cost items ( $\leq$ C30 per item), while the use of medium cost items ( $>$ C70 and $\leq \$ C 350)$ decreased. Duration of surgery and hospital LOS, the only hospital utilisation measures that were available to us, were shorter in the postintervention period but the difference did not differ significantly between the cohorts.

Although we observed a temporal downward trend in costs of disposable supplies after the introduction of our cost-awareness campaign, we cannot assume that the reduction was a direct result of our campaign. The initial campaign itself was less complete than we expected, with none of the 12 participating surgeons attending the potentially most influential part of the campaign, the skills lab providing hands-on experience with the use of reusable laparoscopic devices. Despite that there was a slight increase in the reusable dissectors from only 6 in the control cohort to 14 in the intervention cohort. After the disappointing response to our planned initial campaign, we introduced a second phase which included costawareness OR posters and pocket cards, and the demonstration of reusable devices in the OR coffee lounge. It is possible that the posters, displayed in a variety of OR and other hospital settings, may have had an impact, but we cannot tell whether these minimally invasive interventions actually caused surgeons to change their practice.

A further concern about our study is that the enrolled surgeons would have been aware that their OR costs were being collected after the initial intervention period. The effect of knowing their device use was being observed may have alone influenced their practice to reduce their OR costs. A better design would have been to include a control group of surgeons who were unaware that data were being collected on their OR costs; however, this was beyond the scope of our research.

Several specific changes in surgical practice were identified during our study. The substitution of heated insufflation tubing with much less expensive non-heated tubing, following the publication of a Cochrane Review ${ }^{15}$ that found heated tubing did not produce clinical benefit, and a suture closure device was substituted by a newer standalone barbed suture during the post phase. Although barbed sutures are apparently safe for use in $\mathrm{LH},{ }^{16}$ Health Canada recently published a warning about possible adverse outcomes in bowel surgery, ${ }^{17}$ and this may stimulate further examination of barbed sutures in LH. Another change was the increase in proportion of LH cases in which fallopian tubes were removed, a trend observed by other researchers. This trend is related to emerging evidence that removal of fallopian tubes reduces the risk of developing ovarian cancer. ${ }^{18}$ None of these changes were specifically a focus of our intervention, but they illustrate the types of external evidence that can influence choice of surgical supplies, with possible impact on OR costs.

With an obstetrics and gynaecology resident, we undertook a further study to explore changes in gynaecologists' attitudes towards disposable and non-disposable devices, and to understand how they make decisions to use specific types of device. ${ }^{19}$ This survey was conducted pre and post the cost-awareness campaign. All obstetrician/ gynaecologists associated with the Department of Obstetrics and Gynaecology were included in the survey: those practicing at the hospital and at other Edmonton sites. Our survey found that gynaecologists accorded low priority to cost of devices compared with clinical factors such as effectiveness, safety, ease of use and their own experience. The survey demonstrates that it may be difficult for gynaecologists to adopt cost-awareness into their decision-making.

The strength of our low budget study was the use of a routine OR administrative database supplemented with a small number of variables from patient charts. We found the data remarkably complete. However, as with all routine data, in the case of any anomalous information, particularly in the case of free text variables, there was no way to check specific details. As well, we were dependent on the OR database to identify cases of LH. Few "complications' were mentioned (19 in the precohort and 7 in the postcohort), and there were insufficient details to enable us to relate those events to specific procedures or outcomes. A better research design would have been a prospective study with follow-up after hospital discharge, for example, to postoperative day 30 .

This study has generated many questions. Of most interest would be the relationship between reusable supply costs and disposable supply costs, which was outside the scope of the current study. A different research question, perhaps addressing overall change in OR costs, would be necessary to clarify whether this occurred. In addition, it is possible that procurement activity may have had an impact, for example, if a contract with a specific manufacturer providing generic devices at higher cost was substituted by a contract with another manufacturer providing equivalent but less costly generic devices. We did not observe such changes, but they may have occurred. Other future research topics might also include identifying surgeon-specific strategies that would influence their willingness to include cost-effectiveness information into their decision-making, or focussing on specific groups of devices (such as our middle cost groups) that might be most amenable to switching to lower cost items. A significant limitation of our research is the lack of a control group from another hospital or surgical specialty. Future more rigorous research would increase the validity and utility of studies of cost-awareness interventions for surgeons.

Our simple cost-awareness intervention, incorporating posters and pocket cards (online supplementary appendix 1) would certainly be a generalisable 
intervention based on readily available information. We were able to obtain costs from routine OR data and to model different scenarios reflecting hypothetical practice changes (such as those presented on our posters) and this would be feasible in other hospital settings. We hope that in the future, more comprehensive routine data resources may make the generation of meaningful cost-awareness information available to clinicians in many surgical specialties. As healthcare budgets become more restricted, cost-awareness may have increasing impact on surgeons' decision-making.

\section{CONCLUSION}

Our cost-awareness campaign was temporally associated with a statistically significant reduction in cost of disposable surgical items per case without an increase in shortterm complications. It appears possible that a simple cost-awareness campaign may contribute to cost reduction in the OR by changing surgeon practice, but further detailed analyses are needed to explore whether this is the case and whether this result could be replicated. Our study has raised new research questions about direct and indirect influences on surgeon practice and surgeon attitudes towards cost-awareness and cost containment associated with routine procedures such as LH.

Contributors SR contributed substantially to the conception and design of the study, the acquisition of data, and interpretation of the findings; was involved in all aspects of drafting and revising the paper; has approved the final submitted version of the paper and has agreed to be accountable for all aspects of the work. DL contributed substantially to the conception and design of the study, the acquisition and analysis of data, and interpretation of the findings; was involved in all aspects of drafting and revising the paper; has approved the final submitted version of the paper and has agreed to be accountable for all aspects of the work. GM contributed substantially to the acquisition of data; was involved in revising drafts of the paper; has approved the final submitted version of the paper and has agreed to be accountable for the work. CB contributed substantially to the conception and design of the study, the acquisition of data, and interpretation of the findings; was involved in revising the paper; has approved the final submitted version of the paper and has agreed to be accountable for the work. GR contributed substantially to the conception and design of the study, the acquisition of data, and interpretation of the findings; was involved in aspects of drafting and revising the paper; has approved the final submitted version of the paper and has agreed to be accountable for the work. VAC contributed substantially to the conception and design of the study, and interpretation of the findings; was involved in revising the paper; has approved the final submitted version of the paper and has agreed to be accountable for the work.

Funding This research was funded by the Centre for the Advancement of Minimally Invasive Surgery (CAMIS), Royal Alexandra Hospital. The Cavarzan Chair of Mature Women's Health Research is funded by generous supporters of the Lois Hole Hospital for Women through the Women and Children's Health Research Institute. Neither funder had a role in the conduct of the research or in writing the paper.

Disclaimer The lead author affirms that this manuscript is an honest, accurate and transparent account of the study being reported; that no important aspects of the study have been omitted; and that any discrepancies from the study as planned have been explained.

Competing interests None declared.

Patient consent for publication Not required.

Provenance and peer review Not commissioned; externally peer reviewed.
Data availability statement Data are available upon reasonable request.

Open access This is an open access article distributed in accordance with the Creative Commons Attribution Non Commercial (CC BY-NC 4.0) license, which permits others to distribute, remix, adapt, build upon this work non-commercially, and license their derivative works on different terms, provided the original work is properly cited, appropriate credit is given, any changes made indicated, and the use is non-commercial. See: http://creativecommons.org/licenses/by-nc/4.0/.

ORCID iD

Sue Ross http://orcid.org/0000-0002-0600-4833

\section{REFERENCES}

1 Gray M. A culture of stewardship: the responsibility of NHS leaders to deliver better value healthcare. London, UK NHS Confederation; 2015. https://www.nhsconfed.org/-/media/Confederation/Files/ Publications/Documents/NHS-DoV-Briefing-Document_WEB.pdf

2 Institute of Health Economics/Obrien Institute for Public Health Policy. Physicians as stewards of resources: roles, responsibilities, and remuneration (summary report). Edmonton Institute of Health Economics/Obrien Institute for Public Health Policy; 2016. https:// www.ihe.ca/advanced-search/physicians-as-stewards-of-resourcessummary-report

3 Choosing Wisely Canada. Available: https://choosingwiselycanada. org/

4 Frank JR, Snell L, Sherbino J. CanMEDS 2015 physician competency framework. Ottawa: Royal College of Physicians and Surgeons of Canada, 2015.

5 Long T, Silvestri MT, Dashevsky M, et al. Exit survey of senior residents: cost conscious but uninformed. J Grad Med Educ 2016;8:248-51.

6 Al Zamil MA, Arafa MA. Awareness of surgeons in Saudi Arabia about the surgical costs and investigations: multicenter study. J Surg Educ 2017;74:187-90.

7 Wang A, Dybul SL, Patel PJ, et al. A cross-sectional survey of interventional radiologists and vascular surgeons regarding the cost and reimbursement of common devices and procedures. J Vasc Interv Radiol 2016;27:210-8.

8 Jackson CR, Eavey RD, Francis DO. Surgeon awareness of operating room supply costs. Ann Otol Rhinol Laryngol 2016;125:369-77.

9 Bade K, Hoogerbrug J. Awareness of surgical costs: a multicenter cross-sectional survey. J Surg Educ 2015;72:23-7.

10 Aarts JWM, Nieboer TE, Johnson N, et al. Surgical approach to hysterectomy for benign gynaecological disease. Cochrane Database Syst Rev 2015;194.

11 Childers CP, Showen A, Nuckols T, et al. Interventions to reduce intraoperative costs: a systematic review. Ann Surg 2018;268:48-57.

12 Morgan DM, Kamdar NS, Swenson CW, et al. Nationwide trends in the utilization of and payments for hysterectomy in the United States among commercially insured women. Am J Obstet Gynecol 2018:218:425.e1-425.e18.

13 Lykke R, Blaakær J, Ottesen B, et al. Hysterectomy in Denmark 1977-2011: changes in rate, indications, and hospitalization. Eur $J$ Obstet Gynecol Reprod Biol 2013;171:333-8.

14 Roh HF, Nam SH, Kim JM. Robot-Assisted laparoscopic surgery versus conventional laparoscopic surgery in randomized controlled trials: a systematic review and meta-analysis. PLoS One 2018;13:e0191628.

15 Birch DW, Dang JT, Switzer NJ, et al. Heated insufflation with or without humidification for laparoscopic abdominal surgery. Cochrane Database Syst Rev 2016;11.

16 Tulandi T, Einarsson JI. The use of barbed suture for laparoscopic hysterectomy and myomectomy: a systematic review and metaanalysis. J Minim Invasive Gynecol 2014;21:210-6.

17 Health Canada. Barbed sutures and the risk of small bowel obstruction. Available: http://healthycanadians.gc.ca/recall-alertrappel-avis/hc-sc/2018/66930a-eng.php

18 Dilley SE, Straughn JM, Leath CA. The evolution of and evidence for opportunistic salpingectomy. Obstet Gynecol 2017;130:814-24.

19 Yang H, Capstick VA, Bentz C, et al. Does cost influence the choice of disposable versus reusable instruments? mailed survey of Obstetrician/Gynaecologists. J Obstet Gynaecol Can 2019;41:1416-22. 